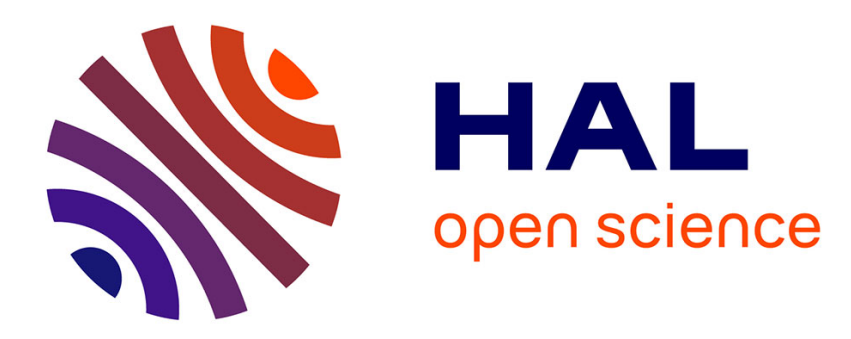

\title{
Toeplitz rectification and DOA estimation with music
}

\author{
P. Vallet, Philippe Loubaton
}

\section{To cite this version:}

P. Vallet, Philippe Loubaton. Toeplitz rectification and DOA estimation with music. ICASSP, May 2014, Firenze, Italy. pp.2237 - 2241, 10.1109/ICASSP.2014.6853997 . hal-01618511

\section{HAL Id: hal-01618511 \\ https://hal.science/hal-01618511}

Submitted on 18 Oct 2017

HAL is a multi-disciplinary open access archive for the deposit and dissemination of scientific research documents, whether they are published or not. The documents may come from teaching and research institutions in France or abroad, or from public or private research centers.
L'archive ouverte pluridisciplinaire HAL, est destinée au dépôt et à la diffusion de documents scientifiques de niveau recherche, publiés ou non, émanant des établissements d'enseignement et de recherche français ou étrangers, des laboratoires publics ou privés. 


\title{
TOEPLITZ RECTIFICATION AND DOA ESTIMATION WITH MUSIC
}

\author{
P. Vallet $^{1}$, P. Loubaton ${ }^{2}$ \\ ${ }^{1}$ Laboratoire IMS (CNRS, Univ. Bordeaux, IPB), 351 Cours de la Libération, 33400 Talence, France \\ ${ }^{2}$ Laboratoire IGM (CNRS, Univ. Paris-Est/MLV), 5 Boulevard Descartes, 77454 Marne-la-Vallée, France \\ pascal.vallet@ipb.fr, loubaton@univ-mlv.fr
}

\begin{abstract}
The MUSIC method is widely used in the field of DoA estimation using an array of $M$ sensors, and is known to perform well as long as the number of available samples $N$ is much larger than $M$. Nevertheless, in the scenario where $N$ is of the same order of magnitude than $M$, its performance degrades, essentially because the sample covariance matrix (SCM) is no more a good estimator. A classical improvement, known as "rectification", consists in forcing the SCM to have a Toeplitz structure. In this paper, we analyze this method, by considering the asymptotic regime where $M, N$ both converge to infinity at the same rate, and by studying consistency and asymptotic normality of the related DoA estimates.
\end{abstract}

Index Terms - DoA estimation, MUSIC, small sample size, Toeplitz rectification.

\section{INTRODUCTION}

The estimation of the directions of arrival (DoA) of source signals using an array of sensors is a fundamental topic in statistical signal processing, which has been extensively studied since the early 1960's. Consider an uniform linear array of $M$ sensors, receiving signals transmitted by $K(K<M)$ narrowband sources, with DoA $\theta_{1}, \ldots, \theta_{K}$. At discrete time $n$, the received sample $\mathbf{y}_{n} \in \mathbb{C}^{M}$ is usually modeled as

$$
\mathbf{y}_{n}=\mathbf{A} \mathbf{s}_{n}+\mathbf{v}_{n}
$$

where $\mathbf{A}=\left[\mathbf{a}\left(\theta_{1}\right), \ldots, \mathbf{a}\left(\theta_{K}\right)\right]$ is the matrix containing the steering vectors $\mathbf{a}\left(\theta_{1}\right), \ldots, \mathbf{a}\left(\theta_{K}\right)$, with $\mathbf{a}(\theta)=M^{-1 / 2}\left[1, \ldots, \mathrm{e}^{\mathrm{i}(M-1) \theta}\right]^{T}$, where $\mathbf{s}_{n} \in \mathbb{C}^{K}$ contains the $K$ source signals received at time $n$, and where $\mathbf{v}_{n}$ is a complex, additive, spatially and temporally white Gaussian noise, with $\mathbb{E}\left[\mathbf{v}_{n} \mathbf{v}_{n}^{*}\right]=\sigma^{2} \mathbf{I}$. Assuming $N$ samples are collected in the matrix $\mathbf{Y}_{N}=\left[\mathbf{y}_{1}, \ldots, \mathbf{y}_{N}\right]$, one obtain

$$
\mathbf{Y}_{N}=\mathbf{A} \mathbf{S}_{N}+\mathbf{V}_{N}
$$

where $\mathbf{S}_{N}=\left[\mathbf{s}_{1}, \ldots, \mathbf{s}_{N}\right]$ et $\mathbf{V}_{N}=\left[\mathbf{v}_{1}, \ldots, \mathbf{v}_{N}\right]$. In the remainder, we assume that the source signals are (unknown) deterministic

Among the popular solutions to the estimation of the DoA $\theta_{1}, \ldots, \theta_{K}$, offering a high resolution performance, the subspace methods, such as MUSIC, are usually prefered over the maximum likelihood based algorithms, due to their reduced computational cost. Under the additional assumption that $\operatorname{rank}\left(\mathbf{S}_{N}\right)=K$, the MUSIC method relies on the fact that $\theta_{1}, \ldots, \theta_{K}$ are zeros of the pseudo-spectrum function

$$
\eta_{N}(\theta)=\mathbf{a}(\theta)^{*} \boldsymbol{\Pi}_{N} \mathbf{a}(\theta),
$$

This work was supported by the French programs GDR ISIS/GRETSI "Jeunes Chercheurs" and ANR-12-MONU-OOO3 DIONISOS Project. where $\boldsymbol{\Pi}_{N}$ is the orthogonal projection matrix onto the kernel of $\mathbf{A S}_{N} \mathbf{S}_{N}^{*} \mathbf{A}^{*}$, i.e. the "noise subspace". Since $\boldsymbol{\Pi}_{N}$ is not available in practice, its standard estimate ${ }^{1} \hat{\boldsymbol{\Pi}}_{N}^{(t)}$ is obtained by computing the orthogonal projection matrix onto the eigenspace associated with the $M-K$ smallest eigenvalues of the sample covariance matrix (SCM)

$$
\frac{\mathbf{Y}_{N} \mathbf{Y}_{N}^{*}}{N}=\frac{1}{N} \sum_{n=1}^{N} \mathbf{y}_{n} \mathbf{y}_{n}^{*},
$$

and the MUSIC algorithm consists in estimating $\theta_{1}, \ldots, \theta_{K}$ as the $K$ most significant minima of

$$
\hat{\eta}_{N}^{(t)}(\theta)=\mathbf{a}(\theta)^{*} \hat{\boldsymbol{\Pi}}_{N}^{(t)} \mathbf{a}(\theta) .
$$

The use of $\hat{\boldsymbol{\Pi}}_{N}$ is motivated by the fact that (law of large number)

$$
\left\|\frac{\mathbf{Y}_{N} \mathbf{Y}_{N}^{*}}{N}-\mathbf{R}_{N}\right\| \rightarrow 0,
$$

almost surely (a.s.), as $M$ is fixed and $N \rightarrow \infty$ (\|. $\|$ stands for the spectral norm), where $\mathbf{R}_{N}=\mathbf{A} \frac{\mathbf{S}_{N} \mathbf{S}_{N}^{*}}{N} \mathbf{A}^{*}+\sigma^{2} \mathbf{I}$ is the covariance matrix of the observations. The convergence (3) implies $\| \hat{\boldsymbol{\Pi}}_{N}^{(t)}-$ $\Pi_{N} \| \rightarrow 0$ and

$$
\sup _{\theta \in[-\pi, \pi]}\left|\hat{\eta}_{N}^{(t)}(\theta)-\eta_{N}(\theta)\right| \underset{N \rightarrow \infty}{\stackrel{a . s .}{\longrightarrow}} 0,
$$

and therefore the consistency of the MUSIC DoA estimates. In practice, the use of the empirical estimate $\hat{\boldsymbol{\Pi}}_{N}^{(t)}$ makes sense whenever $N>>M$, and MUSIC performs relatively well in this context (see Stoica \& Nehorai [1] for a further analysis of the MSE).

However, in certain scenarios, the number of available samples $N$ can be restricted and of the same order of magnitude than the number of sensors $M$, for example when the signals are short-time stationary, or when the array contains a large number of sensors. In this "small sample size" situation, it is well-known that the subspace methods perform very poorly, essentially because the SCM, on which they mainly rely, is no more a good estimator of the true covariance matrix.

To provide a statistical analysis of the previous situation, Mestre [2] proposed to consider a non-standard asymptotic regime in which $M$ and $N$ both converge to infinity at the same rate, that is $M, N \rightarrow$ $\infty$ while $\frac{M}{N} \rightarrow c>0$. In this new asymptotic regime, (3) does not hold anymore, and consequently (2) does not estimate consistently (1). Using results from random matrix theory, Mestre derived an extension of MUSIC (called "G-MUSIC"), in the case of Gaussian temporally i.i.d. signals, by providing a new consistent estimator of any quadratic form $\mathbf{d}_{N}^{*} \boldsymbol{\Pi}_{N} \mathbf{d}_{N}$, with $\left\|\mathbf{d}_{N}\right\|=1$, hence a

\footnotetext{
${ }^{1}$ The superscript $(t)$ stands for traditional estimate.
} 
new consistent estimator of the pseudo-spectrum function. Later, an extension of G-MUSIC, to the case of deterministic unknown signals was derived in [3]. The resulting DoA estimates were shown to outperform the traditional MUSIC estimates, especially for realistic values of $M, N$. However, we notice that the estimator of Mestre does not provide a consistent estimator of the noise subspace projection matrix strictly speaking, that is an estimator $\hat{\boldsymbol{\Pi}}_{N}$ such that $\left\|\hat{\mathbf{\Pi}}_{N}-\boldsymbol{\Pi}_{N}\right\| \rightarrow 0$ a.s., as $M, N \rightarrow \infty, \frac{M}{N} \rightarrow c>0$.

Among the methods to improve the covariance estimation, the so-called Toeplitz rectification is widely used (see e.g. Forster [4] and the references therein), and leads to a remarkable improvement of the DoA estimates, when applied to MUSIC. Under the assumption that $\frac{\mathbf{S}_{N} \mathbf{S}_{N}^{*}}{N}$ converges to a positive diagonal matrix $\boldsymbol{\Gamma}$ (spatial uncorrelation assumption), the covariance matrix $\mathbf{R}_{N}$ converges to the Toeplitz matrix $\mathbf{A} \boldsymbol{\Gamma} \mathbf{A}^{*}+\sigma^{2} \mathbf{I}$, and the Toeplitz rectification consists in forcing the SCM to have a Toeplitz structure by averaging its entries along the diagonals.

In this paper, we propose a statistical analysis of the Toeplitz rectification procedure when $M$ and $N$ converge towards infinity at the same rate. Surprisingly, we prove in section 2 that the rectified SCM is a norm-consistent estimator of the true covariance, in this doubly asymptotic regime decribed above. We also analyze in section 3 the statistical properties of the MUSIC algorithm, when using the rectified SCM, in terms of consistency and asymptotic normality. Finally, in section 4, numerical examples illustrate the proposed results.

To conclude this introduction, we mention that Cai et al. [5] proposed a norm consistent of estimator a large covariance Toeplitz matrix. They used the rectification scheme followed by a tapering of the estimated correlation coefficient, and showed that this procedure leads to a consistent estimator when the entries of the true covariance matrix that are far enough from the main diagonal converge towards 0 fast enough. This hypothesis is of course not verified by the Toeplitz covariance matrices considered here. One of our contribution is to establish that the rectification provides by itself a consistent estimator. We also mention that when $M$ and $N$ are of the same order of magnitude, the spatial smoothing scheme, originally introduced by Evans et al. [6], and studied more recently by Thakre et al. [7], allows in some sense to be back to the case where $M / N$ is small, and to use the standard MUSIC method. We do not discuss this approach here, but mention that the statistical analysis of its performance is a topic of interest.

\section{CONSISTENCY OF THE TOEPLITZ RECTIFICATION}

From now on, we consider the asymptotic regime where $M=$ $M(N)$ is a function of $N$ such that $\frac{M}{N} \rightarrow c>0$ as $N \rightarrow \infty$, and that $K$ is independent of $N$. We moreover consider asymptotically uncorrelated sources in the spatial domain, that is

$$
\frac{\mathbf{S}_{N} \mathbf{S}_{N}^{*}}{N} \underset{N \rightarrow \infty}{\longrightarrow} \boldsymbol{\Gamma}
$$

where $\boldsymbol{\Gamma}$ is a positive diagonal matrix. ${ }^{2}$ In this case, the covariance matrix $\mathbf{R}_{N}$ converge to the Toeplitz matrix $\overline{\mathbf{R}}_{N}=\mathbf{A} \boldsymbol{\Gamma} \mathbf{A}^{*}+\sigma^{2} \mathbf{I}$, that is $\left\|\mathbf{R}_{N}-\overline{\mathbf{R}}_{N}\right\| \rightarrow 0$ as $N \rightarrow \infty$, and the Toeplitz rectification procedure consists in averaging the SCM $\frac{\mathbf{Y}_{N} \mathbf{Y}_{N}^{*}}{N}$ along its diagonals, to obtain a Toeplitz structure. Hence, the $(i, j)$-th entry of the

\footnotetext{
${ }^{2}$ We underline here the fact that the technique used in the forthcoming analysis heavily relies on this source uncorrelation assumption.
}

resulting rectified covariance matrix $\hat{\mathbf{R}}_{N}$ is given by

$$
\left[\hat{\mathbf{R}}_{N}\right]_{i, j}=\frac{1}{M-|i-j|} \sum_{k-l=i-j}\left[\frac{\mathbf{Y}_{N} \mathbf{Y}_{N}^{*}}{N}\right]_{k, l} .
$$

This procedure, also known as "rectification" (or sometimes "Toeplitzification"), can be interpreted as an orthogonal projection onto the space of hermitian Toeplitz matrices [4].

Let $\mathbf{J}$ be the $M \times M$ shift matrix having only non zero entries equal to 1 on the superdiagonal. For a $M \times M$ matrix $\mathbf{X}$, we define by $\mathcal{T}(\mathbf{X})$ the transformation

$$
\mathcal{T}(\mathbf{X})=\sum_{m=-(M-1)}^{M-1} \frac{1}{M-|m|} \operatorname{Tr}\left(\mathbf{X} \mathbf{J}^{m}\right) \mathbf{J}^{-m}
$$

where $\mathbf{J}^{-m}$ stands for $\left(\mathbf{J}^{T}\right)^{m}$, and $\mathbf{J}^{0}=\mathbf{I}$, by abuse of notation. Hence, the rectified SCM can be written in the more appealing way

$$
\hat{\mathbf{R}}_{N}=\mathcal{T}\left(\frac{\mathbf{Y}_{N} \mathbf{Y}_{N}^{*}}{N}\right)
$$

We now analyze the consistency of the rectified SCM $\hat{\mathbf{R}}_{N}$. Surprisingly, the rectified SCM leads to a norm-consistent estimator.

Theorem 1. The following convergence holds

$$
\left\|\hat{\mathbf{R}}_{N}-\mathbf{R}_{N}\right\| \underset{N \rightarrow \infty}{\stackrel{a . s .}{\longrightarrow}} 0 .
$$

Proof. Consider the following bound

$$
\begin{aligned}
&\left\|\hat{\mathbf{R}}_{N}-\mathbf{R}_{N}\right\| \leq\left\|\hat{\mathbf{R}}_{N}-\mathbb{E}\left[\hat{\mathbf{R}}_{N}\right]\right\| \\
&+\left\|\mathbb{E}\left[\hat{\mathbf{R}}_{N}\right]-\left(\mathbf{A} \boldsymbol{\Gamma} \mathbf{A}^{*}+\sigma^{2} \mathbf{I}\right)\right\| \\
&+\left\|\mathbf{A}\left(\frac{\mathbf{S}_{N} \mathbf{S}_{N}^{*}}{N}-\boldsymbol{\Gamma}\right) \mathbf{A}^{*}\right\|,
\end{aligned}
$$

and denote by $\chi_{1, N}, \ldots, \chi_{3, N}$ the three term appearing in the righthand side. Obviously, $\chi_{3, N} \rightarrow 0$ as $N \rightarrow \infty$ from the spatial uncorrelation assumption. Using the classical bound on the spectral norm of hermitian Toeplitz matrices, one have

$$
\chi_{2, N}=\left\|\mathcal{T}\left(\mathbf{A}\left(\frac{\mathbf{S}_{N} \mathbf{S}_{N}^{*}}{N}-\boldsymbol{\Gamma}\right) \mathbf{A}^{*}\right)\right\| \leq \sup _{\nu \in[-\pi, \pi]}\left|g_{N}(\nu)\right|,
$$

where $g_{N}(\nu)$ is the Fourier series of the coefficients defining the Toeplitz matrix $\mathcal{T}\left(\mathbf{A}\left(\frac{\mathbf{s}_{N} \mathbf{S}_{N}^{*}}{N}-\mathbf{\Gamma}\right) \mathbf{A}^{*}\right)$, that is

$$
g_{N}(\nu)=\operatorname{Tr}\left(\mathbf{A}\left(\frac{\mathbf{S}_{N} \mathbf{S}_{N}^{*}}{N}-\boldsymbol{\Gamma}\right) \mathbf{A}^{*} \mathbf{L}(\nu)\right)
$$

where $\mathbf{L}(\nu)$ is defined by

$$
\mathbf{L}(\nu)=\sum_{m=-(M-1)}^{M-1} \frac{\mathrm{e}^{\mathrm{i} m \nu}}{M-|m|} \mathbf{J}^{m} .
$$

Since $\max _{k, l}\left|\mathbf{a}\left(\theta_{k}\right)^{*} \mathbf{L}(\nu) \mathbf{a}\left(\theta_{l}\right)\right| \leq 2$, and $K$ is independent of $N$, we easily deduce that $\chi_{2, N} \rightarrow 0$.

It remains to prove that

$$
\chi_{1, N}=\left\|\mathcal{T}\left(\frac{\mathbf{Y}_{N} \mathbf{Y}_{N}^{*}}{N}-\mathbb{E}\left[\frac{\mathbf{Y}_{N} \mathbf{Y}_{N}^{*}}{N}\right]\right)\right\|
$$


vanishes a.s., as $N \rightarrow \infty$. To that end, we proceed as previously and show that $\sup _{\nu}\left|h_{N}(\nu)\right| \rightarrow 0$, where

$$
h_{N}(\nu)=\operatorname{Tr}\left(\left(\frac{\mathbf{Y}_{N} \mathbf{Y}_{N}^{*}}{N}-\mathbb{E}\left[\frac{\mathbf{Y}_{N} \mathbf{Y}_{N}^{*}}{N}\right]\right) \mathbf{L}(\nu)\right) .
$$

Notice that $h_{N}(\nu)$ can be rewritten in the following way

$h_{N}(\nu)=\mathbf{a}(\nu)^{*}\left(\left(\frac{\mathbf{Y}_{N} \mathbf{Y}_{N}^{*}}{N}-\mathbb{E}\left[\frac{\mathbf{Y}_{N} \mathbf{Y}_{N}^{*}}{N}\right]\right) \odot M \mathbf{L}(0)\right)^{T} \mathbf{a}(\nu)$,

For $\nu_{1}, \nu_{2} \in[-\pi, \pi]$, we have $\left\|\mathbf{a}\left(\nu_{1}\right)-\mathbf{a}\left(\nu_{2}\right)\right\| \leq C N\left|\nu_{1}-\nu_{2}\right|$, where $C>0$ is a constant ${ }^{3}$ independent of $N, \nu_{1}, \nu_{2}$. Using the fact that the spectral norm is sub-multiplicative for the Hadamard product, one obtain that

$$
\begin{aligned}
& \left|h_{N}\left(\nu_{1}\right)-h_{N}\left(\nu_{2}\right)\right| \leq \\
& C N^{2}\left|\nu_{1}-\nu_{2}\right|\left\|\frac{\mathbf{Y}_{N} \mathbf{Y}_{N}^{*}}{N}-\mathbb{E}\left[\frac{\mathbf{Y}_{N} \mathbf{Y}_{N}^{*}}{N}\right]\right\|\|\mathbf{L}(0)\| .
\end{aligned}
$$

Using $\|\mathbf{L}(0)\| \leq\|\mathbf{L}(0)\|_{F}=\mathcal{O}(\sqrt{\log (N)})$, where $\|\cdot\|_{F}$ is the Frobenius norm, and the fact that (see Geman [8])

$$
\left\|\frac{\mathbf{Y}_{N} \mathbf{Y}_{N}^{*}}{N}-\mathbb{E}\left[\frac{\mathbf{Y}_{N} \mathbf{Y}_{N}^{*}}{N}\right]\right\|=\mathcal{O}(1) \text { a.s. }
$$

we get

$$
\left|h\left(\nu_{1}\right)-h\left(\nu_{2}\right)\right| \leq C N^{2} \sqrt{\log (N)}\left|\nu_{1}-\nu_{2}\right| \quad \text { a.s. }
$$

Consider the set $\mathcal{V}_{N}=\left\{\frac{2 \pi k}{N^{3}}-\pi: k=0, \ldots, N^{3}-1\right\}$. For each $\nu \in[-\pi, \pi]$, there exists $\nu_{N} \in \mathcal{V}_{N}$ such that $\left|\nu-\nu_{N}\right|=\mathcal{O}\left(N^{-3}\right)$. In consequence, using (6)

$$
\sup _{\nu \in[-\pi, \pi]}\left|h_{N}(\nu)\right| \leq \max _{\nu \in \mathcal{V}_{N}}|h(\nu)|+\mathcal{O}\left(\frac{\sqrt{\log (N)}}{N}\right) .
$$

Finally, using iteratively a Poincaré inequality for Gaussian variables, as in [9, Lem. 3.1], we can show that for all integer $p$,

$$
\mathbb{E}\left|h_{N}(\nu)\right|^{2 p}=\mathcal{O}\left(\left(\frac{\log (N)}{N}\right)^{p}\right),
$$

which implies, using Markov's inequality, that for all $\epsilon>0$,

$$
\mathbb{P}\left(\max _{\nu \in \mathcal{V}_{N}}\left|h_{N}(\nu)\right| \geq \epsilon\right) \leq \sum_{\nu \in \mathcal{V}_{N}} \mathbb{P}\left(\left|h_{N}(\nu)\right| \geq \epsilon\right)=\mathcal{O}\left(\frac{1}{N^{\ell}}\right)
$$

for all integer $\ell$, and therefore $\chi_{1, N} \rightarrow 0$ a.s.

\section{PERFORMANCE ANALYSIS OF R-MUSIC}

In this section, we provide a statistical analysis in terms of consistency and asymptotic normality of the MUSIC algorithm when using the rectified SCM (refered in the remainder as R-MUSIC).

We thus define a new pseudo-spectrum estimate as

$$
\hat{\eta}_{N}(\theta)=\mathbf{a}(\theta)^{*} \hat{\mathbf{\Pi}}_{N} \mathbf{a}(\theta)
$$

\footnotetext{
${ }^{3}$ We use $C$ as a generic positive constant in the remainder, which may take different values from one line to another.
}

where $\hat{\boldsymbol{\Pi}}_{N}$ is the orthogonal projection matrix onto the eigenspace associated with the $M-K$ smallest eigenvalues of $\hat{\mathbf{R}}_{N}$, Theorem 1 thus implies that

$$
\sup _{\theta \in[-\pi, \pi]}\left|\hat{\eta}_{N}(\theta)-\eta_{N}(\theta)\right| \underset{N \rightarrow \infty}{\stackrel{a . s .}{\longrightarrow}} 0
$$

and the consistency of the related R-MUSIC DoA estimates, which we define formally, for $k=1, \ldots, K$, as

$$
\hat{\theta}_{k, N}=\underset{\theta \in \mathcal{I}_{k}}{\operatorname{argmin}}\left|\hat{\eta}_{N}(\theta)\right|,
$$

where $\mathcal{I}_{1}, \ldots, \mathcal{I}_{k}$ are disjoint compact intervals such that $\theta_{k} \in \mathcal{I}_{k}$. Indeed, we have the following corollary, by applying verbatim the arguments of [9, Prop. 4.1].

Corollary 1. The R-MUSIC DoA estimates satisfy

$$
N\left(\hat{\theta}_{k, N}-\theta_{k}\right) \underset{N \rightarrow \infty}{\stackrel{a . s .}{\longrightarrow}} 0 .
$$

We now study a central limit theorem for the DoA estimates $\hat{\theta}_{1, N}, \ldots, \hat{\theta}_{K, N}$. For this, we define

$$
\rho_{k, N}^{2}=\frac{\sigma^{4} \operatorname{Tr}\left(\mathbf{T}_{k, N}^{2}\right)+2 \sigma^{2} \operatorname{Tr}\left(\mathbf{T}_{k, N}^{2} \mathbf{A} \frac{\mathbf{s}_{N} \mathbf{S}_{N}^{*}}{N} \mathbf{A}^{*}\right)}{\left(\frac{\mathbf{a}^{\prime}\left(\theta_{k}\right)^{*}}{N} \mathbf{\Pi}_{N} \frac{\mathbf{a}^{\prime}\left(\theta_{k}\right)^{*}}{N}\right)^{2}},
$$

with

$$
\begin{aligned}
\mathbf{T}_{k, N}=\frac{1}{2} \mathcal{T}\left(\left(\mathbf{A} \boldsymbol{\Gamma} \mathbf{A}^{*}\right)^{\sharp} \mathbf{a}\left(\theta_{k}\right) \frac{\mathbf{a}^{\prime}\left(\theta_{k}\right)^{*}}{N} \boldsymbol{\Pi}_{N}\right) \\
+\frac{1}{2} \mathcal{T}\left(\boldsymbol{\Pi}_{N} \frac{\mathbf{a}^{\prime}\left(\theta_{k}\right)}{N} \mathbf{a}\left(\theta_{k}\right)^{*}\left(\mathbf{A} \boldsymbol{\Gamma} \mathbf{A}^{*}\right)^{\sharp}\right),
\end{aligned}
$$

where $(.)^{\sharp}$ stands for the pseudo-inverse. Define also

$$
\Delta_{k, N}=\frac{1}{N} \frac{\operatorname{Tr}\left(\mathbf{A}\left(\frac{\mathbf{s}_{N} \mathbf{S}_{N}^{*}}{N}-\boldsymbol{\Gamma}\right) \mathbf{A}^{*} \mathbf{T}_{k, N}\right)}{\frac{\mathbf{a}^{\prime}\left(\theta_{k}\right)^{*}}{N} \boldsymbol{\Pi}_{N} \frac{\mathbf{a}^{\prime}\left(\theta_{k}\right)}{N}} .
$$

Then, we have the following result.

Theorem 2. With the definitions above,

$$
N^{3 / 2} \rho_{k, N}^{-1}\left(\hat{\theta}_{k, N}-\theta_{k}-\Delta_{k, N}\right) \underset{N \rightarrow \infty}{\stackrel{\mathcal{D}}{\longrightarrow}} \mathcal{N}(0,1) .
$$

Proof. Using a Taylor expansion of $\hat{\eta}_{N}^{\prime}(\theta)$ around $\theta_{k}$, we obtain, as in [10],

$$
N^{3 / 2}\left(\hat{\theta}_{k, N}-\theta_{k}\right)=-\sqrt{N} \frac{\operatorname{Re}\left(\frac{\mathbf{a}^{\prime}\left(\theta_{k}\right)^{*}}{N} \hat{\mathbf{\Pi}}_{N} \mathbf{a}\left(\theta_{k}\right)\right)}{\frac{\mathbf{a}^{\prime}\left(\theta_{k}\right)^{*}}{N} \boldsymbol{\Pi}_{N} \frac{\mathbf{a}^{\prime}\left(\theta_{k}\right)}{N}}+o_{\mathbb{P}}(1)
$$

Let $\mathcal{C}$ a circle, counterclockwise oriented, enclosing $\sigma^{2}$ and leaving outside $\gamma_{1}+\sigma^{2}, \ldots, \gamma_{K}+\sigma^{2}$, where $\gamma_{1}, \ldots, \gamma_{K}$ are the diagonal entries of $\boldsymbol{\Gamma}$. Thus from theorem 1, almost surely for all large $N$, $\mathcal{C}$ encloses the $M-K$ smallest eigenvalues of $\hat{\mathbf{R}}_{N}$ and leaves the $K$ largest outside. Moreover, since the $K$ non-zero eigenvalues of A $\boldsymbol{\Gamma} \mathbf{A}^{*}$ converge to $\gamma_{1}, \ldots, \gamma_{K}$, we obtain from residue theorem

$$
\hat{\boldsymbol{\Pi}}_{N}-\boldsymbol{\Pi}_{N}=\frac{1}{2 \pi \mathrm{i}} \oint_{\mathcal{C}}\left(\hat{\mathbf{R}}_{N}-z \mathbf{I}\right)^{-1} \hat{\boldsymbol{\Delta}}_{N}\left(\overline{\mathbf{R}}_{N}-z \mathbf{I}\right)^{-1} \mathrm{~d} z,
$$


where we have denoted $\hat{\boldsymbol{\Delta}}_{N}=\hat{\mathbf{R}}_{N}-\overline{\mathbf{R}}_{N}$ and $\overline{\mathbf{R}}_{N}=\mathbf{A} \boldsymbol{\Gamma} \mathbf{A}^{*}+$ $\sigma^{2} \mathbf{I}$ for short. Replacing $\left(\hat{\mathbf{R}}_{N}-z \mathbf{I}\right)^{-1}$ by $\left(\overline{\mathbf{R}}_{N}-z \mathbf{I}\right)^{-1}$ in the previous integrand, we further obtain

$$
\begin{aligned}
& \hat{\mathbf{\Pi}}_{N}-\boldsymbol{\Pi}_{N}= \\
& \quad \frac{1}{2 \pi \mathrm{i}} \oint_{\mathcal{C}}\left(\overline{\mathbf{R}}_{N}-z \mathbf{I}\right)^{-1} \hat{\boldsymbol{\Delta}}_{N}\left(\overline{\mathbf{R}}_{N}-z \mathbf{I}\right)^{-1} \mathrm{~d} z \\
& -\frac{1}{2 \pi \mathrm{i}} \oint_{\mathcal{C}}\left(\hat{\mathbf{R}}_{N}-z \mathbf{I}\right)^{-1} \hat{\boldsymbol{\Delta}}_{N}\left(\overline{\mathbf{R}}_{N}-z \mathbf{I}\right)^{-1} \hat{\boldsymbol{\Delta}}_{N}\left(\overline{\mathbf{R}}_{N}-z \mathbf{I}\right)^{-1} \mathrm{~d} z .
\end{aligned}
$$

Moreover,

$$
\begin{aligned}
\frac{1}{2 \pi \mathrm{i}} \oint_{\mathcal{C}}\left(\overline{\mathbf{R}}_{N}-z \mathbf{I}\right)^{-1} \hat{\boldsymbol{\Delta}}_{N}\left(\overline{\mathbf{R}}_{N}-z \mathbf{I}\right)^{-1} \mathrm{~d} z= \\
-\boldsymbol{\Pi}_{N} \hat{\boldsymbol{\Delta}}_{N}\left(\mathbf{A} \boldsymbol{\Gamma} \mathbf{A}^{*}\right)^{\sharp}-\left(\mathbf{A} \boldsymbol{\Gamma} \mathbf{A}^{*}\right)^{\sharp} \hat{\boldsymbol{\Delta}}_{N} \boldsymbol{\Pi}_{N} .
\end{aligned}
$$

Let $\mathbf{d}_{1, N}=\boldsymbol{\Pi}_{N} \frac{\mathbf{a}^{\prime}\left(\theta_{k}\right)}{N}$ and $\mathbf{d}_{2, N}=\left(\mathbf{A} \boldsymbol{\Gamma} \mathbf{A}^{*}\right)^{\sharp} \mathbf{a}\left(\theta_{k}\right)$. Straightforward computations leads to

$$
\begin{aligned}
\operatorname{Re}\left(\mathbf{d}_{1, N}^{*}\left(\hat{\mathbf{R}}_{N}-\mathbb{E}\left[\hat{\mathbf{R}}_{N}\right]\right) \mathbf{d}_{2, N}\right)= \\
\operatorname{Tr}\left(\left(\frac{\mathbf{Y}_{N} \mathbf{Y}_{N}^{*}}{N}-\mathbb{E}\left[\frac{\mathbf{Y}_{N} \mathbf{Y}_{N}^{*}}{N}\right]\right) \mathbf{T}_{k, N}\right),
\end{aligned}
$$

and

$$
\mathbb{E}\left|\operatorname{Re}\left(\mathbf{d}_{1, N}^{*}\left(\hat{\mathbf{R}}_{N}-\mathbb{E}\left[\hat{\mathbf{R}}_{N}\right]\right) \mathbf{d}_{2, N}\right)\right|^{2}=N^{-1} \beta_{k, N}^{2},
$$

where $\beta_{k, N}^{2}$ is the denominator of (8). Using the Lyapunov version of the classical central limit theorem, we find

$$
\sqrt{N} \beta_{k, N}^{-1} \operatorname{Re}\left(\mathbf{d}_{1, N}^{*}\left(\hat{\mathbf{R}}_{N}-\mathbb{E}\left[\hat{\mathbf{R}}_{N}\right]\right) \mathbf{d}_{2, N}\right) \underset{N \rightarrow \infty}{\stackrel{\mathcal{D}}{\longrightarrow}} \mathcal{N}(0,1) .
$$

Moreover,

$$
\begin{aligned}
\operatorname{Re}\left(\mathbf{d}_{1, N}^{*}\left(\mathbb{E}\left[\hat{\mathbf{R}}_{N}\right]-\overline{\mathbf{R}}_{N}\right) \mathbf{d}_{2, N}\right)= \\
\operatorname{Tr}\left(\mathbf{A}\left(\frac{\mathbf{S}_{N} \mathbf{S}_{N}^{*}}{N}-\boldsymbol{\Gamma}\right) \mathbf{A}^{*} \mathbf{T}_{k, N}\right) .
\end{aligned}
$$

Thus,

$$
\sqrt{N} \operatorname{Re}\left(\frac{\mathbf{a}^{\prime}\left(\theta_{k}\right)^{*}}{N} \hat{\mathbf{\Pi}}_{N} \mathbf{a}\left(\theta_{k}\right)\right)=\operatorname{Re}\left(\mathbf{d}_{1, N}^{*} \hat{\boldsymbol{\Delta}}_{N} \mathbf{d}_{2, N}\right)+o_{\mathbb{P}}(1)
$$

and the convergence (10) holds.

\section{DISCUSSION AND NUMERICAL RESULTS}

As a first remark on results obtained in the previous section, we notice that the R-MUSIC DoA estimates are consistent from theorem 1 , whatever the level of noise $\sigma^{2}$ is. This is remarkable feature, compared to the G-MUSIC estimates, which are known to be consistent if and only if the SNR is sufficiently high (an explicit condition, called "separation condition" is given in [3]).

However, by analyzing the result of theorem 2, we also remark that the MSE of the R-MUSIC estimates suffers from a"saturation phenomenon". Indeed, it can be proved that the MSE of $\hat{\theta}_{k, N}$ writes

$$
\mathbb{E}\left|\hat{\theta}_{k, N}-\theta_{k}\right|^{2}=\left|\Delta_{k, N}\right|^{2}+\frac{\rho_{k, N}^{2}}{N^{3}}+o\left(\frac{1}{N^{3}}\right),
$$

that is, can be decomposed into a "bias term" $\left|\Delta_{k, N}\right|^{2}$, which is independent of the noise variance $\sigma^{2}$ and mainly depends on the difference between $\frac{\mathbf{S}_{N} \mathbf{S}_{N}^{*}}{N}$ and $\boldsymbol{\Gamma}$, and a "variance term" $N^{-3} \rho_{k, N}^{2}$ decreasing linearly with $\sigma^{2}$. As a consequence, when $\sigma^{2} \rightarrow 0$, the MSE "saturates" at the bias part $\left|\Delta_{k, N}\right|^{2}$. In most practical cases, the difference $\left\|\frac{\mathbf{S}_{N} \mathbf{S}_{N}^{*}}{N}-\Gamma\right\|$ converges to 0 at a rate slower than $\mathcal{O}\left(N^{-1 / 2}\right)$, and the bias part is also preponderant for large $N$.

To summarize, when comparing with the G-MUSIC method, one expects a better behaviour of R-MUSIC in low SNR situations, while in high SNR situations the superiority of G-MUSIC is expected. These remarks are illustrated in figure 1, where we consider an array with $M=20$ sensors, $N=40$ samples and two sources with DoA $\theta_{1}=0$ and $\theta_{2}=0.16$. The SNR is defined as $-10 \log \left(\sigma^{2}\right)$. The entries of the source signal matrix $\mathbf{S}_{N}$ are the realization of standard independent Gaussian variables. The prepon-

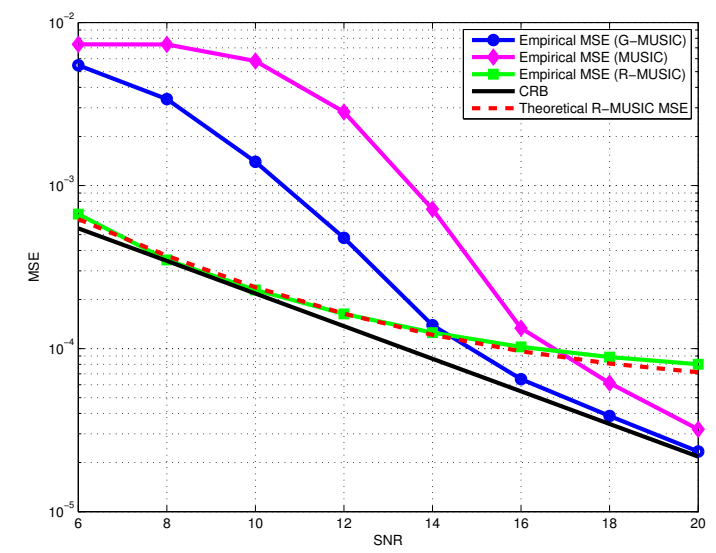

Fig. 1. MSE saturation with Gaussian uncorrelated signals

derance of the bias part of the MSE is visible from $14 \mathrm{~dB}$, while the G-MUSIC achieves the Cramer-Rao bound around $16 \mathrm{~dB}$.

In figure 2, we consider the same parameters, except that the source signals are generated as mutually independent AR(1) processes, with correlation 0.9 . We observe in this case that the bias is more important than for the Gaussian temporally uncorrelated signals.

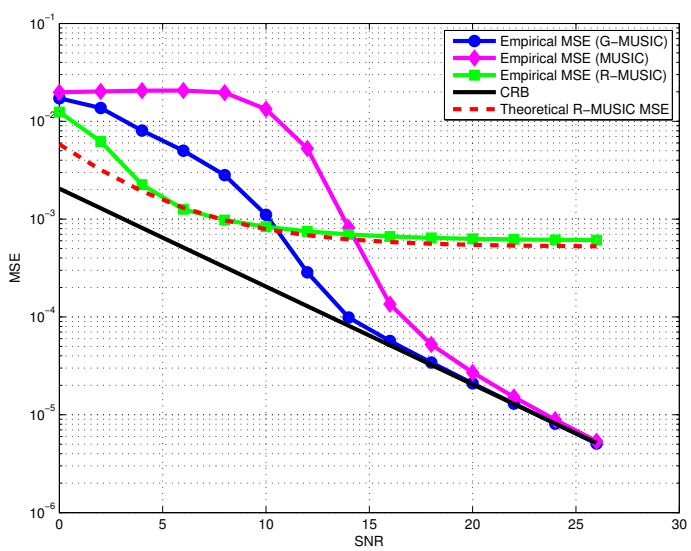

Fig. 2. MSE saturation with Gaussian AR(1) signals 


\section{REFERENCES}

[1] P. Stoica and A. Nehorai, "Music, maximum likelihood, and cramer-rao bound," IEEE Trans. Acoust., Signal Proc., vol. 37, no. 5, pp. 720-741, 1989.

[2] X. Mestre, "Improved estimation of eigenvalues and eigenvectors of covariance matrices using their sample estimates," IEEE Trans. Inf. Theory, vol. 54, no. 11, pp. 5113-5129, 2008.

[3] P. Vallet, P. Loubaton, and X. Mestre, "Improved subspace estimation for multivariate observations of high dimension: The deterministic signal case," IEEE Trans. Inf. Theory, vol. 58, no. 2, Feb. 2012.

[4] P. Forster, "Generalized rectification of cross spectral matrices for arrays of arbitrary geometry," IEEE Trans. Signal Process., vol. 49, no. 5, pp. 972-978, 2001.

[5] T. Tony Cai, Zhao Ren, and Harrison H. Zhou, "Optimal rates of convergence for estimating Toeplitz covariance matrices," Probab. Theory Related Fields, vol. 156, no. 1-2, pp. 101-143, 2013.

[6] J. Evans, J. Johnson, and D. Sun, "High resolution angular spectrum estimation techniques for terrain scattering analysis and angle of arrival estimation," in Proc. 1st ASSP Workshop Spectral Estimation, 1981, pp. 134-139.

[7] A. Thakre, M. Haardt, and K. Giridhar, "Single snapshot spatial smoothing with improved effective array aperture," IEEE Signal Processing Letters, vol. 16, no. 6, pp. 505-508, 2009.

[8] S. Geman, "A limit theorem for the norm of random matrices," Ann. Prob., pp. 252-261, 1980.

[9] W. Hachem, P. Loubaton, X. Mestre, J. Najim, and P. Vallet, "Large information plus noise random matrix models and consistent subspace estimation in large sensor networks," Random Matrices: Theory Appl., vol. 1, no. 2, 2012.

[10] P. Vallet, X. Mestre, and P. Loubaton, "A clt for the g-music doa estimator," in Proceedings of the 20th European Signal Processing Conference (EUSIPCO). IEEE, 2012, pp. 22982302. 